\title{
New applications of cardiovascular magnetic resonance to guide cardiac resynchronization therapy
}

\author{
Joanna Petryka ${ }^{1,2}$, Artur Oręziak ${ }^{3}$, Andrzej Przybylski ${ }^{3}$, Jolanta Miśko² \\ 1Department of Coronary Artery Disease and Structural Heart Diseases, Institute of Cardiology, Warsaw, Poland \\ 2Magnetic Resonance Unit, Institute of Cardiology, Warsaw, Poland \\ ${ }^{3}$ Department of Arrhythmia, Institute of Cardiology, Warsaw, Poland
}

Postep Kardiol Inter 2012; 8, 3 (29): 234-238

DOI: $10.5114 /$ pwki.2012.30403

Key words: cardiovascular magnetic resonance, resynchronization therapy, delayed enhancement, right ventricle

\section{Introduction}

Cardiac resynchronization therapy (CRT) is a treatment of proven efficacy in patients with heart failure, impaired left ventricular ejection fraction and wide QRS complex. It has been demonstrated that CRT has a good effect on left ventricular remodelling, and improves outcome and quality of life. However, in $1 / 3$ of patients this therapy brings no expected benefit. There are many variables that may influence the response to resynchronization described in the literature. Among others, the presence of mechanical dyssynchrony of the left ventricle and the amount of myocardial fibrosis assessed with cardiovascular magnetic resonance (CMR) have been mentioned [1]. The assessment of left ventricular contraction dyssynchrony in CMR is based on postprocessing multi-stage data, which makes this method difficult to use in clinical practice. In contrast, evaluation of delayed enhancement (DE), which corresponds with myocardial fibrosis, is a relatively simple and well-documented technique. The CMR study with fibrosis imaging guides the procedure of left ventricular lead placement to avoid the scar. Furthermore, the assessment of the scar enables one to evaluate the amount of viable left ventricular myocardium ready to undertake synchronised contractile activity after CRT implantation. Along with the dynamic development of magnetic resonance imaging technique and better understanding of its clinical usefulness, new applications of CMR in patients referred for CRT have been reported. Apart from previously mentioned methods of dyssynchrony and delayed enhancement assessment, the growing role of evaluation of right ventricular function and scar homogeneity has been shown.

\section{Assessment of right ventricle}

The interest of scientists in multi-centre randomized clinical trials on patients with CRT has been mainly focused on the assessment of left ventricular systolic function prior to device implantation and the evaluation of left ventricular remodelling as a result of the therapy. Therefore the guidelines of the European Society of Cardiology, which are based on the data from clinical trials, contain only the criterion of left ventricular ejection fraction. Whereas right ventricular dysfunction and its impact on the response to CRT are not fully documented.

The presence of systolic dysfunction of the right ventricle is a strong and independent predictor of death in patients with chronic heart failure [2, 3]. The results of studies on limited patient populations suggest an improvement in functional and volumetric parameters of the right ventricle after implantation of the CRT device [4, 5], while the improvement of systolic function of both ventricles leads to better patient outcome $[6,7]$. There have been papers published in recent years documenting the impact of baseline assessment of right ventricular systolic function on the response to resynchronization therapy [8-10]. Significant impairment of right ventricular function may limit the beneficial remodelling of the left ventricle after therapy. However, the interdependence of left and right ventricular contractility and the reason for a different degree of improvement for each ventricle in patients after CRT remain not fully described.

Because of the anatomy and complex geometry, the role of echocardiographic and scintigraphic methods in evaluation of the right ventricle is limited. Cardiovascu-

\section{Corresponding author:}

Dr Joanna Petryka, Department of Coronary Artery Disease and Structural Heart Disease, Institute of Cardiology, 42 Alpejska, 04-628 Warsaw,

Poland, e-mail: joannapetryka@hotmail.com

Praca wpłynęła: 25.07.2012, przyjęta do druku: 27.07.2012. 
lar magnetic resonance on the other hand provides imaging of the right ventricle in a freely chosen view (Figure 1) and the assessment of its volumetric and functional parameters in a 3D model (Figure 2), which guarantees better precision and reproducibility of the results [11]. In a study published last year [12] it was demonstrated that right ventricular dysfunction assessed with cardiovascular magnetic resonance was associated with a lack of response to resynchronization therapy and with more frequent occurrence of cardiovascular events including deaths and hospital admissions. It should be noted that there was a wide range of the degree of right ventricular systolic function impairment in patients undergoing CRT, whereas the degree of left ventricular function impairment was relatively homogeneous as it was one of the criteria for the therapy. Therefore, right ventricular ejection fraction was an independent factor distinguishing this population. Lower ejection
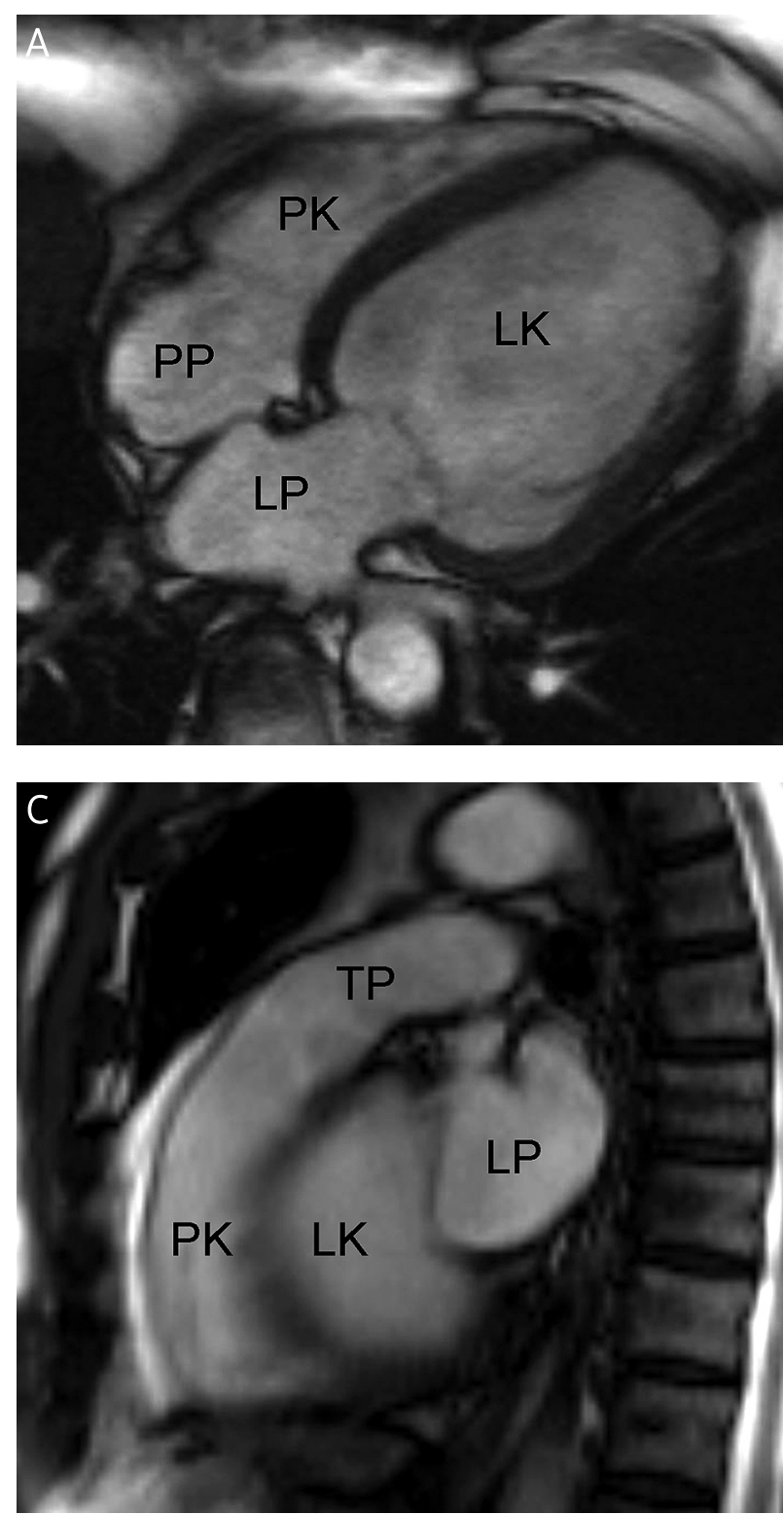

fraction of the right ventricle was associated with lower ejection fraction of the left ventricle, higher significance of mitral regurgitation and higher mean pulmonary artery pressure. The authors suggest that the right ventricular dysfunction arises in this population in two main mechanisms: either as a result of biventricular impairment of myocardial contractility or as a result of pulmonary hypertension secondary to elevated left ventricular filling pressure and mitral regurgitation. Among patients with the ejection fraction of the right ventricle below $30 \%$, only $20 \%$ of subjects fulfilled the criteria of a response to resynchronization therapy.

The disadvantage of cardiovascular magnetic resonance is a lack of possibility to routinely perform serial assessment of the right ventricle in patients with previously implanted devices. However, the evaluation of the right ventricle should become a routine element of complex CMR

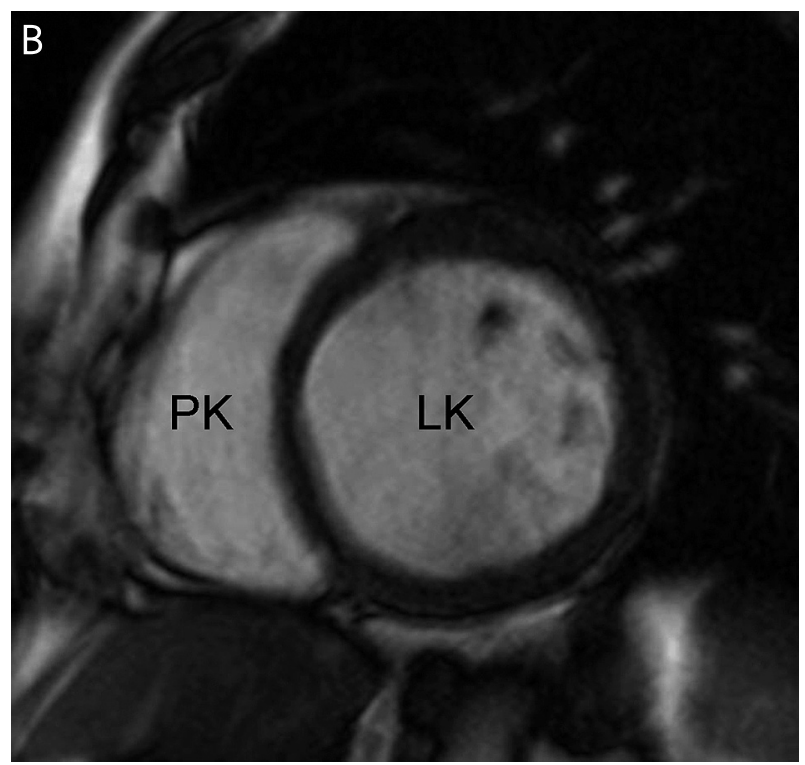

Fig. 1. Standard projection for visualisation of right ventricle in cardiac magnetic resonance $(A-$ four chamber view, B - short axis view, C - right ventricular outflow tract)

$L K$ - left ventricle, $L P$ - left atrium, $P K$ - right ventricle, $P P$ - right atrium, TP - pulmonary main artery 


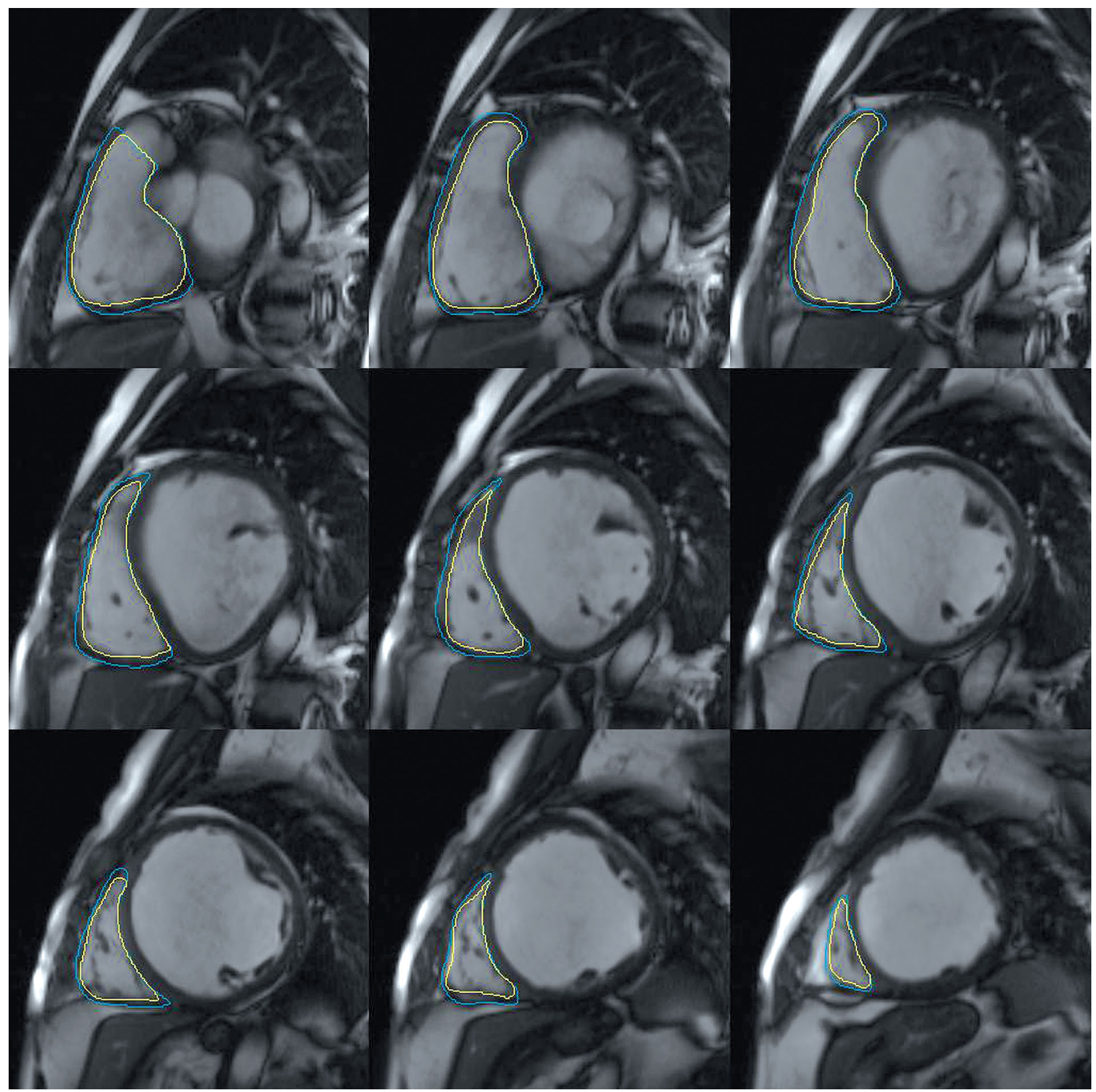

Fig. 2. Assessment of right ventricular systolic function in 3D model based on short axis slices from base to apex. The endocardial and epicardial borders are delineated semi-automatically in order to obtain the following parameters: end-diastolic volume, end-systolic volume, stroke volume, ejection fraction and mass of right ventricle

assessment of patients referred for CRT as assessment of delayed enhancement.

\section{Arrhythmia and delayed gadolinium enhancement}

Most of the patients referred for CRT fulfil the criteria for cardioverter-defibrillator (ICD) implantation. Therefore, a significant percentage of implanted resynchronisation devices, around $70-80 \%$, are also equipped with a defibrillator (CRT-D) [13]. However, the advantage of CRT-D over
CRT-P (cardiac resynchronization therapy with pacemaker) with regards to the outcome of patients with an implanted resynchronization device is not well documented. It has been proven that implantation of a CRT-P device leads to the limitation of ventricular arrhythmia, reduces the risk of sudden cardiac death and improves the outcome [6]. Also the number of adequate cardioverter-defibrillator interventions is relatively low in this patient group [14]. Furthermore, CRT-D implantation is more expensive and associated with additional risk of complications, including 

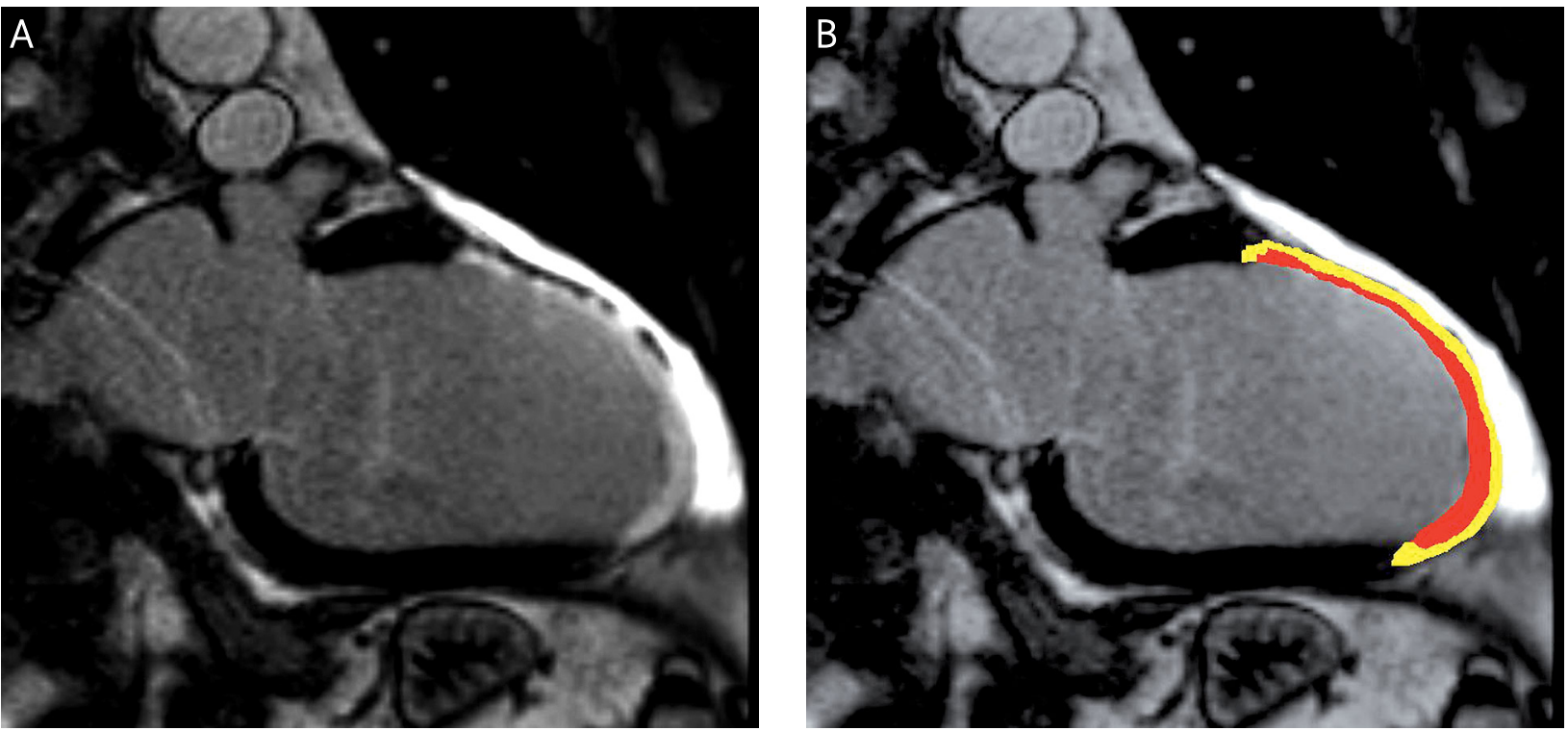

Fig. 3. Delayed enhancement of myocardium in cardiac magnetic resonance (A - two chamber long axis view with visualized delayed enhancement corresponding with the scar in mid and apical segments of the anterior wall and in the apex of the left ventricle, B - diagram showing differentiation of the scar into two zones: in red - core of the scar of the highest signal; in yellow - borderline zone between the core and normal myocardium corresponding with grey zone)

inadequate ICD interventions, than in the case of CRT-P. It is thus essential to develop algorithms identifying patients with a high risk of sudden cardiac death in whom the benefit from CRT-D would be maximized.

Cardiovascular magnetic resonance with the use of delayed gadolinium enhancement provides the evaluation of myocardial structure and identification of fibrotic tissue (Figure 3). It has been suggested that the assessment of scar extent and its heterogeneity in CMR enables the stratification of the risk of arrhythmia in patients after myocardial infarction [15]. So far the published studies indicate the key role of the zone of intermediate degree of fibrosis between the normal myocardium and the central part of the scar - the grey zone/border zone [15-18]. A greater amount of grey zone in the scar was associated with worse outcome and more frequent ventricular arrhythmia in patients with coronary artery disease. Recently, a study assessing the presence of scar with its components in CMR in patients referred for resynchronization therapy has been published [19]. It was found that the analysis of delayed enhancement can be applied to identify patients of low risk of ventricular arrhythmia. Those patients are characterised by a grey zone of smaller extent and lower percentage of scarred myocardium or totally viable myocardium in CMR. Furthermore, the presence of a homogeneous scar, that is a scar with a smaller grey zone, was associated with less frequent arrhythmia occurrence than the presence of a scar with a high percentage of myocardium of borderline degree of fibrosis. Interestingly, in the cited paper the extent of delayed gadolinium enhancement was a predictor of ven- tricular arrhythmia and adequate ICD intervention independently of the aetiology of heart failure. If those results are confirmed in further studies, in the future the analysis of the extent of delayed gadolinium enhancement and certain components of the scar in CMR could enable identification of patients at low risk of malignant ventricular arrhythmia, in whom ICD implantation together with a resynchronization device would not bring additional benefits.

\section{Conclusions}

The key to maximise the benefit from resynchronization therapy is adequate patient selection and optimal performance of the CRT implantation procedure. Cardiovascular magnetic resonance may turn out to be a useful tool to guide optimal left ventricular lead placement, to identify high-risk patients due to right ventricular systolic dysfunction, and to support the clinical decision between CRT-D and CRT-P devices.

\section{References}

1. Petryka J, Miśko J, Przybylski A, et al. Magnetic resonance imaging assessment of intraventricular dyssynchrony and delayed enhancement as predictors of response to cardiac resynchronization therapy in patients with heart failure of ischaemic and non-ischaemic etiologies. Eur J Radiol 2011 Nov 4.

2. de Groote P, Millaire A, Foucher-Hossein C, et al. Right ventricular ejection fraction is an independent predictor of survival in patients with moderate heart failure. J Am Coll Cardiol 1998; 32: 948-954.

3. Ghio S, Gavazzi A, Campana C, et al. Independent and additive prognostic value of right ventricular systolic function and 
pulmonary artery pressure in patients with chronic heart failure. J Am Coll Cardiol 2001; 37: 183-188.

4. Bleeker GB, Schalij MJ, Nihoyannopoulos P, et al. Left ventricular dyssynchrony predicts right ventricular remodeling after cardiac resynchronization therapy. J Am Coll Cardiol 2005; 46: 2264-2269.

5. Rajagopalan N, Suffoletto MS, Tanabe M, et al. Right ventricular function following cardiac resynchronization therapy. Am J Cardiol 2007; 100: 1434-1436.

6. Cleland JG, Daubert JC, Erdmann E, et al. The effect of cardiac resynchronization on morbidity and mortality in heart failure. N Engl J Med 2005; 352: 1539-1549.

7. Ypenburg C, Van Bommel RJ, Borleffs CJ, et al. Long-term prognosis after cardiac resynchronization therapy is related to the extent of left ventricular reverse remodeling at midterm follow-up. J Am Coll Cardiol 2009; 53: 483-490.

8. Field ME, Solomon SD, Lewis EF, et al. Right ventricular dysfunction and adverse outcome in patients with advanced heart failure. J Card Fail 2006; 12: 616-620.

9. Scuteri L, Rordorf R, Marsan NA, et al. Relevance of echocardiographic evaluation of right ventricular function in patients undergoing cardiac resynchronization therapy. Pacing Clin Electrophysiol 2009; 32: 1040-1049.

10. Tabereaux PB, Doppalapudi H, Kay GN, et al. Limited response to cardiac resynchronization therapy in patients with concomitant right ventricular dysfunction. J Cardiovasc Electrophysiol 2010; 21: 431-435.

11. Hudsmith LE, Petersen SE, Francis JM, et al. Normal human left and right ventricular and left atrial dimensions using steady state free precession magnetic resonance imaging. J Cardiovasc Magn Reson 2005; 7: 775-782.

12. Alpendurada F, Guha K, Sharma R, et al. Right ventricular dysfunction is a predictor of non-response and clinical outcome following cardiac resynchronization therapy. J Cardiovasc Magn Reson 2011; 13: 68.

13. Dickstein K, Bogale N, Priori S, et al. The European cardiac resynchronization therapy survey. Eur Heart J 2009; 30: 2450-2460.

14. Saxon LA, Bristow MR, Boehmer J, et al. Predictors of sudden cardiac death and appropriate shock in the comparison of medical therapy, pacing, and defibrillation in heart failure (COMPANION) trial. Circulation 2006; 114: 2766-2772.

15. Roes SD, Borleffs CJW, van der Geest RJ, et al. Infarct tissue heterogeneity assessed with contrast-enhanced MRI predicts spontaneous ventricular arrhythmia in patients with ischemic cardiomyopathy and implantable cardioverter-defibrillator. Circ Cardiovasc Imaging 2009; 2: 183-190.

16. Yan AT, Shayne AJ, Brown KA, et al. Characterization of the periinfarct zone by contrast-enhanced cardiac magnetic resonance imaging is a powerful predictor of post-myocardial infarction mortality. Circulation 2006; 114: 32-39.

17. Schmidt A, Azevedo CF, Cheng A, et al. Infarct tissue heterogeneity by magnetic resonance imaging identifies enhanced cardiac arrhythmia susceptibility in patients with left ventricular dysfunction. Circulation 2007; 115: 2006-2014.

18. Heidary S, Patel H, Chung J, et al. Quantitative tissue characterization of infarct core and border zone in patients with ischemic cardiomyopathy by magnetic resonance is associated with future cardiovascular events. J Am Coll Cardiol 2010; 55: 2762-2768.

19. Fernández-Armenta J, Berruezo A, Mont L, et al. Use of myocardial scar characterization to predict ventricular arrhythmia in cardiac resynchronization therapy. Circ Arrhythm Electrophysiol 2012; 5: 111-121. 